\title{
Deciphering weak decays of triply heavy baryons by SU(3) analysis
}

\author{
Fei Huang $^{2, a}$, Ji Xu ${ }^{1, b}$, Xi-Ruo Zhang ${ }^{1}$ \\ ${ }^{1}$ School of Physics and Microelectronics, Zhengzhou University, Zhengzhou 450001, Henan, China \\ 2 INPAC, Key Laboratory for Particle Astrophysics and Cosmology (MOE), Shanghai Key Laboratory for Particle Physics and Cosmology, \\ School of Physics and Astronomy, Shanghai Jiao Tong University, Shanghai 200240, China
}

Received: 6 August 2021 / Accepted: 7 October 2021 / Published online: 6 November 2021

(C) The Author(s) 2021

\begin{abstract}
Baryons with three heavy quarks are the last missing pieces of the lowest-lying baryon multiplets in the quark model after the discovery of doubly heavy baryons. In this work, we study nonleptonic weak decays of triply heavy baryons $\Omega_{c c c}^{++}, \Omega_{b b b}^{-}, \Omega_{c c b}^{+}$, and $\Omega_{c b b}^{0}$. Decay amplitudes for various processes have been parametrized in terms of the $\mathrm{SU}(3)$ irreducible nonperturbative amplitudes. A number of relations for the partial decay widths can be deduced from these results that can be examined in future. Some decay channels and cascade decay modes which likely to be used to reconstruct the triply heavy baryons have been also listed.
\end{abstract}

\section{Introduction}

Triply heavy baryons which consist of three heavy $c$ or $b$ quarks are of great theoretical interests since they refrain from light quark contaminations. Being baryonic analogues of heavy quarkonium, the study of triply heavy baryons can help us to better understand the dynamics of strong interactions and would yield sharp tests for QCD. Besides, these baryons also provide particular information on the three body static potential. Previous studies on triply heavy baryons mainly concentrated on spectroscopy, relevant theoretical tools such as nonrelativistic constituent quark model (NRCQM) [1], potential nonrelativistic QCD (pNRQCD) [2], and the QCD sum rule (QCDSR) [3] have been developed to investigate the nature of these baryons.

In the past decades, hadron spectroscopy has experienced a continuous progress. Since 2016, the BESIII Collaboration has reanalyzed the singly charmed baryon decays with higher precision $[4,5]$. One milestone for the doubly charmed baryon spectroscopy is the discovery of $\Xi_{c c}^{++}$by the LHCb Collaboration [6,7]. Afterwards, baryons with three heavy quarks are the last missing pieces of the lowest-lying baryon

\footnotetext{
a e-mail: fhuang@sjtu.edu.cn

be-mail: xuji_phy@zzu.edu.cn (corresponding author)
}

multiplets in quark model, with this in mind, it is timely and meaningful to analyze triply heavy baryon on both theoretical and experimental sides at this stage. The flavor SU(5) group includes all types of baryons containing zero, one, two or three heavy quarks. One should note that the differences of quark masses break the flavor symmetry, the larger the group, the bigger the amount of breaking. The masses of three light quarks $u, d, s$ are much smaller than the masses of $c$ and $b$ quarks, this makes the flavor $\mathrm{SU}(3)$ symmetry basically maintained in weak decays of heavy baryons. However, the SU(5) group algebra helps us identify the triply heavy baryons which are interested in this paper. As an example, two sets of corresponding baryons projected along the $u, d, s, c$ and $u, d, s, b$ quarks are depicted in Fig. 1 .

The production of triply heavy baryons is difficult and no experimental signal for any of them has been observed yet. The production rate of triply heavy charmed baryon in $e^{+} e^{-}$ collision has been estimated to be very small [8], however, a recent investigation finds that around $10^{4}-10^{5}$ events of triply heavy baryons can be accumulated for $10 \mathrm{fb}^{-1}$ integrated luminosity at LHC [9]. The heavy quarks can be produced via $g g$ fusion and quark-antiquark annihilation at hadron colliders. LHC and the future high luminosity LHC provide us a good chance to discover these triply heavy baryons. LHC has helped us find out doubly heavy baryon, undoubtedly, it will provide a sustained progress in heavy baryon field as well as the breadth and depth necessary for a vibrant research environment.

Many studies about the triply heavy baryons can be found in the literature [9-21], however, despite the great progress, little attention has been paid to the decay properties. Various types of weak decays of triply heavy baryons occur, but unfortunately, a universal dynamical (factorization) approach has not be established yet. There are several distinct energy scales involved in the weak decays of triply heavy baryons which make the systematic factorization unavailable at present, these are the mass $m$ of heavy $c$ or $b$ quark, the momen- 
Fig. 1 Baryons with spin 3/2 made from four quarks of the types $u, d, s, c$ (a) and $u, d, s, b$ (b). Triply heavy baryons $\Omega_{c c c}^{++}$ and $\Omega_{b b b}^{-}$are localized in the highest layers

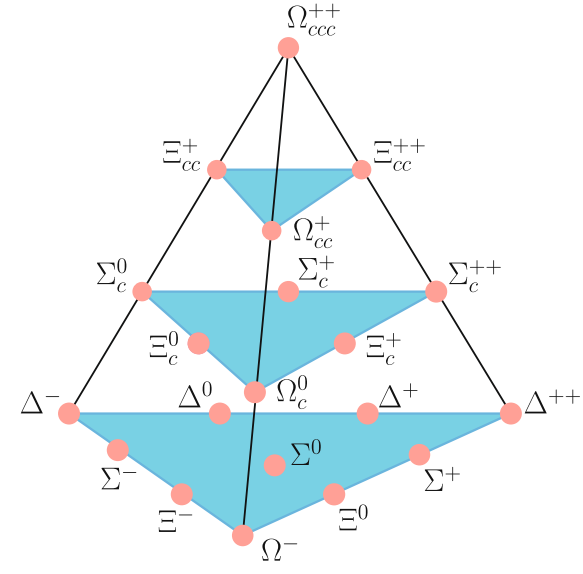

a

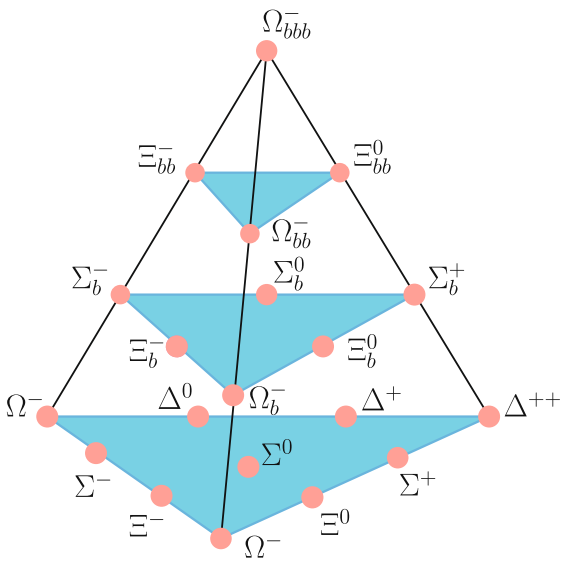

b tum of the heavy quark $m v$, the off-shell energy of the heavy quark $m v^{2}$, and the energy of light hadron in the final state. This poses an obstacle for us to predict the decay width of triply heavy baryons. On the other hand, the approach of flavor SU(3) symmetry allows us to relate decay modes in the $b$ and $c$-hadron decays despite the unknown non-perturbative dynamics of QCD [22-48]. In this work, we consider nonleptonic decay channels of triply heavy baryons by utilizing flavor SU(3) analysis, it is an extension and supplement of a series of previous works. Reference [46] has discussed semileptonic and nonleptonic decay modes of $\Omega_{c c c}^{++}, \Omega_{b b b}^{-}$, $\Omega_{c c b}^{+}$and $\Omega_{b b c}^{0}$. Beyond these modes, several two-body and some three-body decay which are not covered in previous work would be considered in this work. The experimental data of the doubly heavy baryon $\Xi_{c c}^{++}$indicated that the decay modes of this particle are not saturated by two-body intermediate states. One may naturally expect this would happen to the triply heavy baryons, some CKM allowed three-body decay processes of $\Omega_{c c c}^{++}$considered in this paper might be helpful for finding this particle in future. Certain particular interesting signature modes of triply heavy baryon decays such as $\Omega_{c c c}^{++} \rightarrow \Omega_{s s s}^{-}+3 \pi^{+}$will be discussed in the frame of SU(3) analysis. The main motivation of this work is to provide some suggestions which may help experimentalists find triply heavy baryons in future.

The present manuscript is arranged as follows. In Sect. 2, we present the irreducible forms of baryon and meson states under flavor SU(3) symmetry. In Sect. 3, nonleptonic decays of triply charmed baryon $\Omega_{c c c}^{++}$, triply bottom baryon $\Omega_{b b b}^{-}$, the mixed triply heavy baryons $\Omega_{c c b}^{+}$and $\Omega_{b b c}^{0}$ will be studied in order. A short summary is given in the last section.

\section{Particle multiplets}

In this section, we will collect the representations for hadron multiplets under the flavor SU(3) group. The best determi- nation of the magnitudes of CKM matrix elements and the Cabibbo parametrization will be also presented.

We start with the baryon sector. The initial triply heavy baryon singlet is given by

$$
\left(\Omega_{c c c}^{++}\right),\left(\Omega_{b b b}^{-}\right), \quad\left(\Omega_{c c b}^{+}\right), \quad\left(\Omega_{b b c}^{0}\right) .
$$

The doubly heavy baryons are an SU(3) triplet:

$T_{c c}=\left(\begin{array}{c}\Xi_{c c}^{++} \\ \Xi_{c c}^{+} \\ \Omega_{c c}^{+}\end{array}\right), \quad T_{b c}=\left(\begin{array}{c}\Xi_{b c}^{+} \\ \Xi_{b c}^{0} \\ \Omega_{b c}^{0}\end{array}\right), \quad T_{b b}=\left(\begin{array}{c}\Xi_{b b}^{0} \\ \Xi_{b b}^{-} \\ \Omega_{b b}^{-}\end{array}\right)$.

Singly charmed baryons with two light quarks can form an antitriplet or sextet which are

$$
\begin{aligned}
T_{c \overline{3}}= & \left(\begin{array}{ccc}
0 & \Lambda_{c}^{+} & \Xi_{c}^{+} \\
-\Lambda_{c}^{+} & 0 & \Xi_{c}^{0} \\
-\Xi_{c}^{+} & -\Xi_{c}^{0} & 0
\end{array}\right) \\
T_{\mathbf{c 6}}= & \left(\begin{array}{ccc}
\Sigma_{c}^{++} & \frac{1}{\sqrt{2}} \Sigma_{c}^{+} & \frac{1}{\sqrt{2}} \Xi_{c}^{\prime+} \\
\frac{1}{\sqrt{2}} \Sigma_{c}^{+} & \Sigma_{c}^{0} & \frac{1}{\sqrt{2}} \Xi_{c}^{\prime 0} \\
\frac{1}{\sqrt{2}} \Xi_{c}^{\prime+} & \frac{1}{\sqrt{2}} \Xi_{c}^{\prime 0} & \Omega_{c}^{0}
\end{array}\right) .
\end{aligned}
$$

Light baryons made of three light quarks can group into an SU(3) octet and a decuplet. The octet has the expression:

$T_{8}=\left(\begin{array}{ccc}\frac{1}{\sqrt{2}} \Sigma^{0}+\frac{1}{\sqrt{6}} \Lambda^{0} & \Sigma^{+} & p \\ \Sigma^{-} & -\frac{1}{\sqrt{2}} \Sigma^{0}+\frac{1}{\sqrt{6}} \Lambda^{0} & n \\ \Xi^{-} & \Xi^{0} & -\sqrt{\frac{2}{3}} \Lambda^{0}\end{array}\right)$.

The indices of the decuplet is symmetric, it can be written in a compact form, 


$$
\begin{aligned}
T_{10}= & \frac{1}{\sqrt{3}}\left(\left(\begin{array}{ccc}
\sqrt{3} \Delta^{++} & \Delta^{+} & \Sigma^{\prime+} \\
\Delta^{+} & \Delta^{0} & \frac{\Sigma^{\prime}}{\sqrt{2}} \\
\Sigma^{\prime+} & \frac{\Sigma^{\prime 0}}{\sqrt{2}} & \Xi^{\prime 0}
\end{array}\right),\left(\begin{array}{ccc}
\Delta^{+} & \Delta^{0} & \frac{\Sigma^{\prime 0}}{\sqrt{2}} \\
\Delta^{0} & \sqrt{3} \Delta^{-} & \Sigma^{\prime-} \\
\frac{\Sigma^{0}}{\sqrt{2}} & \Sigma^{\prime-} & \Xi^{\prime-}
\end{array}\right)\right. \\
& \left.\times\left(\begin{array}{ccc}
\Sigma^{\prime+} & \frac{\Sigma^{\prime 0}}{\sqrt{2}} & \Xi^{\prime 0} \\
\frac{\Sigma^{\prime 0}}{\sqrt{2}} & \Sigma^{\prime-} & \Xi^{\prime-} \\
\Xi^{\prime 0} & \Xi^{\prime-} & \sqrt{3} \Omega^{-}
\end{array}\right)\right)
\end{aligned}
$$

For the meson sector, the light pseudoscalar mesons form an octet:

$M_{8}=\left(\begin{array}{ccc}\frac{\pi^{0}}{\sqrt{2}}+\frac{\eta_{8}}{\sqrt{6}} & \pi^{+} & K^{+} \\ \pi^{-} & -\frac{\pi^{0}}{\sqrt{2}}+\frac{\eta}{\sqrt{6}} & K^{0} \\ K^{-} & \bar{K}^{0} & -2 \frac{\eta_{8}}{\sqrt{6}}\end{array}\right)$.

We note that the $\eta$ in our calculations is only considered as a member of octet, while the singlet $\eta_{1}$ is not considered here to avoid the octet-singlet mixture complexity. The charmed and bottom mesons form similar SU(3) antitriplet,

$D_{i}=\left(D^{0}, D^{+}, D_{s}^{+}\right), \quad B_{i}=\left(B^{-}, \bar{B}^{0}, \bar{B}_{s}^{0}\right)$.

Here we also present the best determination of the magnitudes of the CKM matrix elements [49] matrix elements. Tree operators of charm-quark decays into light quarks are categorized into three groups: Cabibboallowed, singly Cabibbo-suppressed, and doubly Cabibbosuppressed,

$c \rightarrow s \bar{d} u, \quad c \rightarrow u \bar{d} d / \bar{s} s, \quad c \rightarrow d \bar{s} u$.

These tree operators transform under the flavor SU(3) symmetry as $\mathbf{3} \otimes \overline{\mathbf{3}} \otimes \mathbf{3}=\mathbf{3} \oplus \mathbf{3} \oplus \overline{\mathbf{6}} \oplus \mathbf{1 5}$. Thus the effective Hamiltonian can be decomposed in terms of a vector $\mathrm{H}_{3}$; a traceless tensor antisymmetric in upper indices, $\mathrm{H}_{\overline{6}}$; a traceless tensor symmetric in upper indices, $H_{15}$. The representation $\mathrm{H}_{3}$ will vanish as an approximation by taking $V_{c d}^{*} V_{u d}=-V_{c s}^{*} V_{u s} \simeq-\sin \theta_{c}$ [44]. The nonzero components of hadron-level Hamiltonian are listed below:

$$
\left(H_{\overline{6}}\right)_{2}^{31}=-\left(H_{\overline{6}}\right)_{2}^{13}=1, \quad\left(H_{15}\right)_{2}^{31}=\left(H_{15}\right)_{2}^{13}=1,
$$

Cabibbo allowed;

$$
\left(H_{\overline{6}}\right)_{3}^{31}=-\left(H_{\overline{6}}\right)_{3}^{13}=\left(H_{\overline{6}}\right)_{2}^{12}=-\left(H_{\overline{6}}\right)_{2}^{21}=\sin \theta_{c},
$$

Singly Cabibbo suppressed ;

$\left(H_{15}\right)_{3}^{31}=\left(H_{15}\right)_{3}^{13}=-\left(H_{15}\right)_{2}^{12}=-\left(H_{15}\right)_{2}^{21}=\sin \theta_{c}$,

Singly Cabibbo suppressed ;

$\left(H_{\overline{6}}\right)_{3}^{21}=-\left(H_{\overline{6}}\right)_{3}^{12}=\sin ^{2} \theta_{c}, \quad\left(H_{15}\right)_{3}^{21}=\left(H_{15}\right)_{3}^{12}=\sin ^{2} \theta_{c}$,

Doubly Cabibbo suppressed .

$$
\left[\begin{array}{ccc}
\left|V_{u d}\right| & \left|V_{u s}\right| & \left|V_{u b}\right| \\
\left|V_{c d}\right| & \left|V_{c s}\right| & \left|V_{c b}\right| \\
\left|V_{t d}\right| & \left|V_{t s}\right| & \left|V_{t b}\right|
\end{array}\right]=\left[\begin{array}{ccc}
0.97370 \pm 0.00014 & 0.2245 \pm 0.0008 & 0.00382 \pm 0.00024 \\
0.221 \pm 0.004 & 0.987 \pm 0.011 & 0.0410 \pm 0.0014 \\
0.0080 \pm 0.0003 & 0.0388 \pm 0.0011 & 1.013 \pm 0.030
\end{array}\right]
$$

and the Cabibbo parametrization formalism

$\left[\begin{array}{ll}V_{u d} & V_{u s} \\ V_{c d} & V_{c s}\end{array}\right]=\left[\begin{array}{cc}\cos \theta_{c} & \sin \theta_{c} \\ -\sin \theta_{c} & \cos \theta_{c}\end{array}\right]$,

to make the following discussions more comprehensible.

To depict the processes of various decay modes under the frame of flavor SU(3) analysis, we need to construct the hadron-level effective Hamiltonian in addition to the representations for initial and final states which have been listed above. It is necessary to point out that a hadron in the final state must be created by its antiparticle field. For instance, for a $\Sigma_{c}^{++}$appearing in the final state, we need the $\overline{\Sigma_{c}^{++}}$in the Hamiltonian. The construction of hadron-level effective Hamiltonian will shown in the next section.

\section{Nonleptonic decays of triply heavy baryons}

\subsection{Nonleptonic $\Omega_{c c c}^{++}$decays}

We start with the nonleptonic $\Omega_{c c c}^{++}$decays. We have neglected penguin contributions in charm-quark decays since they are highly suppressed by the relevant CKM
For $\Omega_{c c c}^{++}$decays into two $D$-mesons and a light baryon, the corresponding Hamiltonian can be constructed as

$$
\begin{aligned}
\mathcal{H}_{e f f}= & a_{1} \Omega_{c c c}^{++} \varepsilon_{i j k}\left(\bar{T}_{8}\right)_{l}^{k} \bar{D}^{i} \bar{D}^{m}\left(H_{\overline{6}}\right)_{m}^{i l} \\
& +a_{2} \Omega_{c c c}^{++} \varepsilon_{i j k}\left(\bar{T}_{8}\right)_{l}^{k} \bar{D}^{l} \bar{D}^{m}\left(H_{\overline{6}}\right)_{m}^{i j} \\
& +a_{3} \Omega_{c c c}^{++} \varepsilon_{i j k}\left(\bar{T}_{8}\right)_{l}^{k} \bar{D}^{i} \bar{D}^{m}\left(H_{15}\right)_{m}^{i l} \\
& +a_{4} \Omega_{c c c}^{++}\left(\bar{T}_{10}\right)_{i j k} \bar{D}^{k} \bar{D}^{l}\left(H_{15}\right)_{l}^{i j} .
\end{aligned}
$$

Where the $a_{i}$ 's are $\mathrm{SU}(3)$ irreducible nonperturbative amplitudes. The first three terms in Eq. (12) denote the light baryon containing in the final states belongs to SU(3) octet, the last term denotes the light baryon in SU(3) decuplet. Feynman diagrams for these modes are given in Fig. 2. Decay amplitudes for various channels can be deduced from the Hamiltonian in Eq. (12), and are collected in Table 1 (light baryon in octet) and Table 2 (light baryon in decuplet).

A few remarks are given in order:

1. The initial state $\Omega_{c c c}^{++}$is flavor $\mathrm{SU}(3)$ singlet, thus has no index to connect to the final states and $H_{\overline{6}, 15}$, this is a unique property comparing with previous works on weak decays of singly and doubly heavy baryons. 


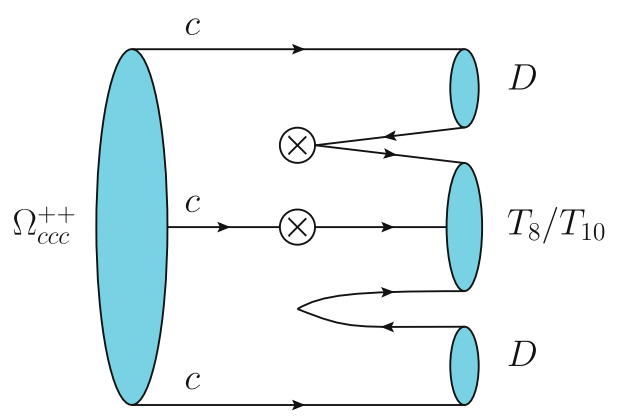

Fig. 2 The Feynman diagrams for $\Omega_{c c c}^{++}$decays into two $D$-mesons and a light baryon (octet or decuplet)

2. Tables 1 and 2 are arranged according to the decay amplitude's dependence on $\sin \theta_{c}, c \rightarrow s$ transition is proportional to $V_{c s} \sim 1$, while $c \rightarrow d$ transition has a Cabibbo suppressed CKM matrix element $V_{c s} \sim 0.2$.

3. A number of relations for decay widths can be readily deduced from Tables 1 and 2,

$$
\begin{aligned}
& \Gamma\left(\Omega_{c c c}^{++} \rightarrow D^{0} D_{s}^{+} \Sigma^{+}\right)=\Gamma\left(\Omega_{c c c}^{++} \rightarrow D^{+} D^{0} p\right), \\
& \Gamma\left(\Omega_{c c c}^{++} \rightarrow D^{+} D^{+} n\right)=\Gamma\left(\Omega_{c c c}^{++} \rightarrow D_{s}^{+} D_{s}^{+} \Xi^{0}\right), \\
& \Gamma\left(\Omega_{c c c}^{++} \rightarrow D^{0} D^{+} \Sigma^{+}\right)=\Gamma\left(\Omega_{c c c}^{++} \rightarrow D^{+} D^{+} \Sigma^{0}\right), \\
& \Gamma\left(\Omega_{c c c}^{++} \rightarrow D^{+} D_{s}^{+} \Lambda^{0}\right)=\frac{1}{3} \Gamma\left(\Omega_{c c c}^{++} \rightarrow D_{s}^{+} D^{+} \Sigma^{0}\right), \\
& \Gamma\left(\Omega_{c c c}^{++} \rightarrow D^{0} D^{+} \Sigma^{\prime+}\right)=\Gamma\left(\Omega_{c c c}^{++} \rightarrow D_{s}^{+} D^{+} \Xi^{\prime 0}\right) \\
& =\Gamma\left(\Omega_{c c c}^{++} \rightarrow D^{+} D^{+} \Sigma^{\prime 0}\right),
\end{aligned}
$$

$$
\begin{aligned}
& \Gamma\left(\Omega_{c c c}^{++} \rightarrow D^{0} D_{s}^{+} \Delta^{+}\right)=\Gamma\left(\Omega_{c c c}^{++} \rightarrow D_{s}^{+} D^{+} \Delta^{0}\right) \\
& =\Gamma\left(\Omega_{c c c}^{++} \rightarrow D_{s}^{+} D_{s}^{+} \Sigma^{\prime 0}\right), \\
& \Gamma\left(\Omega_{c c c}^{++} \rightarrow D^{0} D^{+} \Delta^{+}\right)=\Gamma\left(\Omega_{c c c}^{++} \rightarrow D^{0} D_{s}^{+} \Sigma^{\prime+}\right) \\
& =\frac{1}{2} \Gamma\left(\Omega_{c c c}^{++} \rightarrow D^{+} D^{+} \Delta^{0}\right)=\frac{1}{2} \Gamma\left(\Omega_{c c c}^{++} \rightarrow D_{s}^{+} D_{s}^{+} \Xi^{\prime 0}\right) .
\end{aligned}
$$

4. Current researches about weak decays of doubly heavy baryon concentrate on two-body modes and there are few studies on decay modes of triply heavy baryon [50-56]. In terms of quasi two-body decay approach as well as the idea of quark-diquark correspondence, the transition $\Omega_{c c c}^{++} \rightarrow T_{8 / 10} D D$ might be related to $B_{c}^{-} \rightarrow D^{*} \bar{D} \rightarrow M D \bar{D}$. For $B_{c}^{-} \rightarrow D^{*} \bar{D}$, the branching ratio may reach several percent and most of $D^{*}$ would decay to $D$-meson plus a light meson $[49,57]$. Therefore $\mathcal{B R}\left(B_{c}^{-} \rightarrow M D \bar{D}\right)$ may reach percent level. Take the smallness of $V_{c b}$ into consideration, it is plausible to expect $\mathcal{B R}\left(\Omega_{c c c}^{++} \rightarrow T_{8 / 10} D D\right)$ at the same level.

However, it is necessary to point out that the above relationships between decay widths are obtained in the flavor SU(3) symmetry limit, in which the mass differences between final state hadrons have been ignored. Although the influence of identical particles on phase space integration has been considered, these relationships will be modified when calculating the kinematic corrections. Besides, the hadronization processes whose information contained in different decay con-
Table 1 Amplitudes for $\Omega_{c c c}^{++}$ decays into two $D$-mesons and a light baryon (octet)

Table 2 Amplitudes for $\Omega_{c c c}^{++}$ decays into two $D$-mesons and a light baryon (decuplet)

\begin{tabular}{llll}
\hline Channel & Amplitude & Channel & Amplitude \\
\hline$\Omega_{c c c}^{++} \rightarrow D^{0} D^{+} \Sigma^{+}$ & $-a_{1}+2 a_{2}-a_{3}$ & $\Omega_{c c c}^{++} \rightarrow D^{+} D^{+} \Lambda^{0}$ & $\sqrt{\frac{2}{3}}\left(-a_{1}+2 a_{2}+3 a_{3}\right)$ \\
$\Omega_{c c c}^{++} \rightarrow D^{+} D^{+} \Sigma^{0}$ & $\sqrt{2}\left(a_{1}-2 a_{2}+a_{3}\right)$ & $\Omega_{c c c}^{++} \rightarrow D^{+} D_{s}^{+} \Xi^{0}$ & $-a_{1}+2 a_{2}+a_{3}$ \\
$\Omega_{c c c}^{++} \rightarrow D^{0} D^{+} p$ & $\left(a_{1}-2 a_{2}+a_{3}\right)\left(-\sin \theta_{c}\right)$ & $\Omega_{c c c}^{++} \rightarrow D^{0} D_{s}^{+} \Sigma^{+}$ & $\left(a_{1}-2 a_{2}+a_{3}\right)\left(-\sin \theta_{c}\right)$ \\
$\Omega_{c c c}^{++} \rightarrow D^{+} D^{+} n$ & $2\left(-a_{1}+2 a_{2}+a_{3}\right) \sin \theta_{c}$ & $\Omega_{c c c}^{++} \rightarrow D^{+} D_{s}^{+} \Lambda^{0}$ & $\frac{\left(a_{1}-2 a_{2}+3 a_{3}\right)}{\sqrt{6}} \sin \theta_{c}$ \\
$\Omega_{c c c}^{++} \rightarrow D^{+} D_{s}^{+} \Sigma^{0}$ & $\frac{\left(a_{1}-2 a_{2}+3 a_{3}\right)}{\sqrt{2}} \sin \theta_{c}$ & $\Omega_{c c c}^{++} \rightarrow D_{s}^{+} D_{s}^{+} \Xi^{0}$ & $2\left(-a_{1}+2 a_{2}+a_{3}\right) \sin \theta_{c}$ \\
$\Omega_{c c c}^{++} \rightarrow D^{0} D_{s}^{+} p$ & $\left(a_{1}-2 a_{2}+a_{3}\right) \sin ^{2} \theta_{c}$ & $\Omega_{c c c}^{++} \rightarrow D^{+} D_{s}^{+} n$ & $\left(a_{1}-2 a_{2}-a_{3}\right) \sin ^{2} \theta_{c}$ \\
$\Omega_{c c c}^{++} \rightarrow D_{s}^{+} D_{s}^{+} \Lambda^{0}$ & $-\sqrt{\frac{8}{3}}\left(a_{1}-2 a_{2}\right) \sin ^{2} \theta_{c}$ & $\Omega_{c c c}^{++} \rightarrow D_{s}^{+} D_{s}^{+} \Sigma^{0}$ & $-2 \sqrt{2} a_{3} \sin ^{2} \theta_{c}$ \\
\hline
\end{tabular}

\begin{tabular}{llll}
\hline Channel & Amplitude & Channel & Amplitude \\
\hline$\Omega_{c c c}^{++} \rightarrow D^{0} D^{+} \Sigma^{\prime+}$ & $\frac{2 a_{4}}{\sqrt{3}}$ & $\Omega_{c c c}^{++} \rightarrow D^{+} D^{+} \Sigma^{\prime 0}$ & $\sqrt{\frac{8}{3}} a_{4}$ \\
$\Omega_{c c c}^{++} \rightarrow D_{s}^{+} D^{+} \Xi^{\prime 0}$ & $\frac{22 a_{4}}{\sqrt{3}}$ & & \\
$\Omega_{c c c}^{++} \rightarrow D^{0} D^{+} \Delta^{+}$ & $-\frac{2 a_{4}}{\sqrt{3}} \sin \theta_{c}$ & $\Omega_{c c c}^{++} \rightarrow D^{0} D_{s}^{+} \Sigma^{\prime+}$ & $\frac{2 a_{4}}{\sqrt{3}} \sin \theta_{c}$ \\
$\Omega_{c c}^{++} \rightarrow D^{+} D^{+} \Delta^{0}$ & $-\frac{4 a_{4}}{\sqrt{3}} \sin \theta_{c}$ & $\Omega_{c c}^{++} \rightarrow D_{s}^{+} D_{s}^{+} \Xi^{\prime 0}$ & $\frac{4 a_{4}}{\sqrt{3}} \sin \theta_{c}$ \\
$\Omega_{c c c}^{++} \rightarrow D^{0} D_{s}^{+} \Delta^{+}$ & $\frac{2 a_{4}}{\sqrt{3}} \sin ^{2} \theta_{c}$ & $\Omega_{c c c}^{++} \rightarrow D_{s}^{+} D^{+} \Delta^{0}$ & $\frac{2 a_{4}}{\sqrt{3}} \sin ^{2} \theta_{c}$ \\
$\Omega_{c c c}^{++} \rightarrow D_{s}^{+} D_{s}^{+} \Sigma^{\prime 0}$ & $\sqrt{\frac{8}{3}} a_{4} \sin ^{2} \theta_{c}$ & & \\
\hline
\end{tabular}


stants and form factors would also affect the relationships derived in this paper. Once the mass of $\Omega_{c c c}^{++}$is experimentally measured in the future, a rigorous analysis would be necessary.

Particular decay processes of $\Omega_{c c c}^{++}$in the detectors can be used as signatures to reconstruct this triply heavy baryon. The ground states of triply heavy baryons can decay only through the weak interaction. An interesting decay mode has been proposed by Ref. [9]:

$\Omega_{c c c}^{++} \rightarrow \Omega_{c c s}^{+}+\pi^{+} \rightarrow \Omega_{c s s}^{0}+2 \pi^{+} \rightarrow \Omega_{s s s}^{-}+3 \pi^{+}$.

With this cascade mode, $\Omega_{c c c}^{++}$finally decays to $\Omega_{c c c}^{++}+3 \pi^{+}$ and every step is Cabibbo-allowed. Having the results of previous works at hand $[19,44,46]$, we can write down other Cabibbo-allowed cascade modes of $\Omega_{c c c}^{++}$which might be useful for finding this triply heavy baryon. They are collected in Table 3. All the processes shown in this table can be categorized into color-allowed external $W$-emission, colorsuppressed internal $W$-emission and sum of these two. The most color-favored cascade decay mode has been presented in Eq. (14), other relatively color-favored processes are

$$
\begin{aligned}
& \Omega_{c c c}^{++} \rightarrow \Xi_{c c}^{++}+\bar{K}^{0} \rightarrow \Xi_{c}^{\prime+} / \Xi_{c}^{+}+\bar{K}^{0}+\pi^{+} \\
& \rightarrow \Xi^{\prime 0} / \Xi^{0}+2 \pi^{+}+\bar{K}^{0}, \\
& \Omega_{c c c}^{++} \rightarrow \Omega_{c c s}^{+}+\pi^{+} \rightarrow \Omega_{c s s}^{0}+2 \pi^{+} \rightarrow \Xi^{\prime 0} / \Xi^{0} \\
& +2 \pi^{+}+\bar{K}^{0}, \\
& \Omega_{c c c}^{++} \rightarrow \Omega_{c c s}^{+}+\pi^{+} \rightarrow \Xi_{c}^{\prime+} / \Xi_{c}^{+}+\pi^{+} \\
& +\bar{K}^{0} \rightarrow \Xi^{\prime 0} / \Xi^{0}+2 \pi^{+}+\bar{K}^{0} .
\end{aligned}
$$

\subsection{Nonleptonic $\Omega_{b b b}^{-}$decays}

For the bottom-quark decay, we can categorize the quarklevel transitions into four kinds,

$$
\begin{array}{rlrl}
b & \rightarrow c \bar{c} d / s, & b & \rightarrow c \bar{u} d / s, \\
b \rightarrow u \bar{c} d / s, & b \rightarrow q \bar{q} q .
\end{array}
$$

We will study $\Omega_{b b b}^{-} \rightarrow T_{b c} B_{c}$ and $\Omega_{b b b}^{-} \rightarrow T_{b c} B_{c} M$ for the first quark-level transition $b \rightarrow c \bar{c} d / s$ case. For the second $b \rightarrow c \bar{u} d / s$ case, decay modes $\Omega_{b b b}^{-} \rightarrow T_{b c} B$, $\Omega_{b b b}^{-} \rightarrow T_{b c} B M$ and $\Omega_{b b b}^{-} \rightarrow B B T_{c \overline{3} / 6}$ will be discussed. The $b \rightarrow u \bar{c} d / s$ is highly suppressed by the corresponding CKM matrix elements as illustrated by Eq. (8), therefore it is not considered in this paper. For the last $b \rightarrow q \bar{q} q$ case, the decay modes $\Omega_{b b b}^{-} \rightarrow B B T_{8 / 10}$ would be analyzed.

The transition operator $b \rightarrow c \bar{c} d / s$ can form an SU(3) triplet with $\left(H_{3}\right)_{2}=V_{c d}^{*}$ and $\left(H_{3}\right)_{3}=V_{c s}^{*}$, one has the

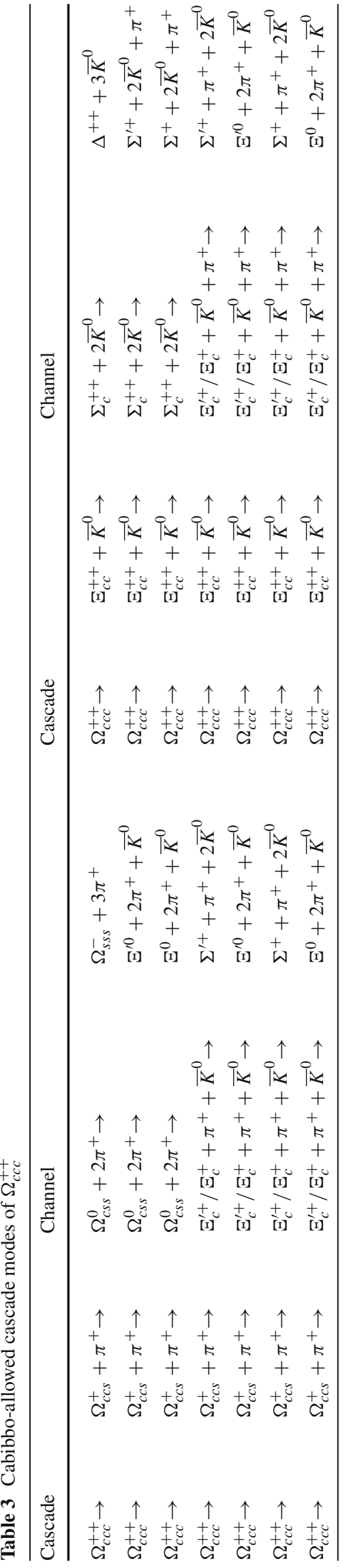


Fig. 3 Feynman diagrams for $\Omega_{b b b}^{-}$decays into a doubly heavy baryon $T_{b c}$, plus a $B_{c}$ meson and a light meson
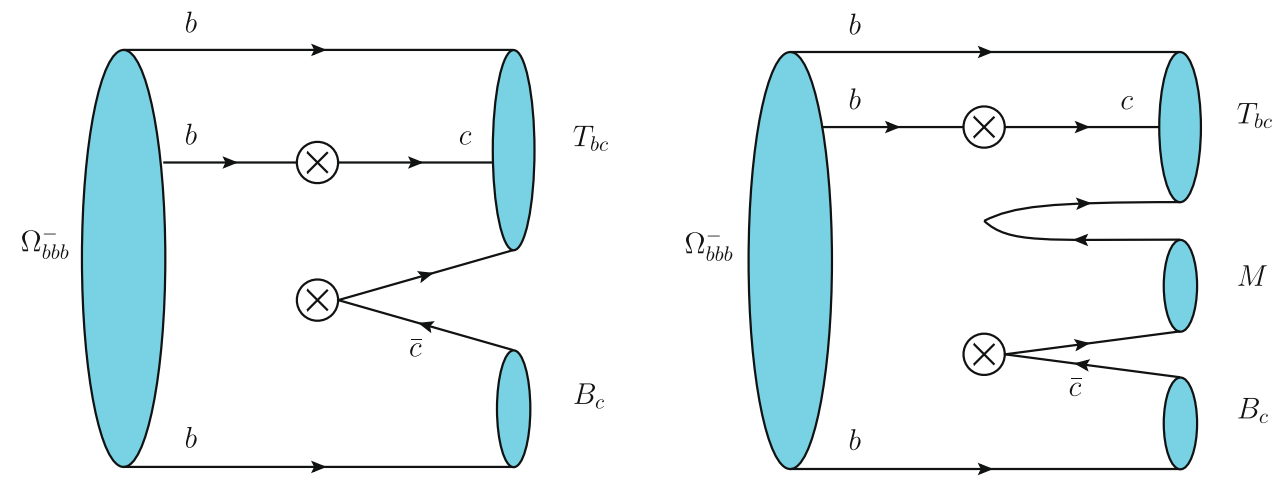

Table 4 Amplitudes for $\Omega_{b b b}$ decays into $T_{b c}$ and a $B_{c}$ meson plus a light meson

\begin{tabular}{llll}
\hline Channel & Amplitude & Channel & Amplitude \\
\hline$\Omega_{b b b}^{-} \rightarrow \Xi_{b c}^{0} B_{c}$ & $b_{1} V_{c b} V_{c s}^{*}$ & & \\
$\Omega_{b b b}^{-} \rightarrow \Xi_{b}^{+} \pi^{-} B_{c}$ & $b_{2} V_{c b} V_{c s}^{*}$ & $\Omega_{b b b}^{-} \rightarrow \Xi_{b c}^{0} \pi^{0} B_{c}$ & $-\frac{b_{2} V_{c b} V_{c s}^{*}}{\sqrt{2}}$ \\
$\Omega_{b b b}^{-} \rightarrow \Xi_{b c}^{0} \eta B_{c}$ & $\frac{b_{2} V_{c b} V_{c s}^{*}}{\sqrt{6}}$ & $\Omega_{b b b}^{-} \rightarrow \Omega_{b c}^{0} K^{0} B_{c}$ & $b_{2} V_{c b} V_{c s}^{*}$ \\
\hline
\end{tabular}

Hamiltonian for $\Omega_{b b b}^{-}$decays into a doubly heavy baryon $T_{b c}$, plus a $B_{c}$ and a light meson:

$\mathcal{H}_{\text {eff }}=b_{1} \Omega_{b b b}^{-}\left(\bar{T}_{b c}\right)_{i} B_{c}\left(H_{3}\right)^{i}+b_{2} \Omega_{b b b}^{-}\left(\bar{T}_{b c}\right)_{i} B_{c} M_{j}^{i}\left(H_{3}\right)^{j}$.

The relevant Feynman diagrams are presented in Fig. 3. Decay amplitudes for different channels are obtained by expanding the above Hamiltonian and are collected in Table 4. These lead to the relations for decay widths:

$$
\begin{aligned}
& \Gamma\left(\Omega_{b b b}^{-} \rightarrow \Xi_{b c}^{0} \pi^{0} B_{c}\right)=\frac{1}{2} \Gamma\left(\Omega_{b b b}^{-} \rightarrow \Xi_{b c}^{+} \pi^{-} B_{c}\right) \\
& =\frac{1}{2} \Gamma\left(\Omega_{b b b}^{-} \rightarrow \Omega_{b c}^{0} K^{0} B_{c}\right)=3 \Gamma\left(\Omega_{b b b}^{-} \rightarrow \Xi_{b c}^{0} \eta B_{c}\right) .
\end{aligned}
$$

The transition operator $b \rightarrow c \bar{u} d / s$ forms an SU(3) octet $H_{8}$ with nonzero entries

$$
\left(H_{8}\right)_{1}^{2}=V_{c b} V_{u d}^{*}, \quad\left(H_{8}\right)_{1}^{3}=V_{c b} V_{u s}^{*} .
$$

Thus, we have the effective Hamiltonian for $\Omega_{b b b}^{-}$decays into a doubly heavy baryon $T_{b c}$, plus a $B$-meson and a light meson:

$$
\begin{aligned}
\mathcal{H}_{e f f}= & c_{1} \Omega_{b b b}^{-}\left(\bar{T}_{b c}\right)_{i} \bar{B}^{j}\left(H_{8}\right)_{j}^{i}+c_{2} \Omega_{b b b}^{-}\left(\bar{T}_{b c}\right)_{i} \bar{B}^{i} M_{k}^{j}\left(H_{8}\right)_{j}^{k} \\
& +c_{3} \Omega_{b b b}^{-}\left(\bar{T}_{b c}\right)_{i} \bar{B}^{j} M_{k}^{i}\left(H_{8}\right)_{j}^{k} \\
& +c_{4} \Omega_{b b b}^{-}\left(\bar{T}_{b c}\right)_{i} \bar{B}^{j} M_{j}^{k}\left(H_{8}\right)_{k}^{i} .
\end{aligned}
$$

The Feynman diagrams for these decays modes are given in Fig. 4. Expanding the above equations, we will obtain the decay amplitudes given in Table 5, which lead to one relation for decay widths:

$$
\Gamma\left(\Omega_{b b b}^{-} \rightarrow \Omega_{b c}^{0} B^{-} \pi^{0}\right)=\frac{1}{2} \Gamma\left(\Omega_{b b b}^{-} \rightarrow \Omega_{b c}^{0} \bar{B}^{0} \pi^{-}\right) .
$$

The quark-level transition operator for $\Omega_{b b b}^{-}$decays into two $B$-mesons and one singly charmed baryon (antitriplet or sextex) is also $b \rightarrow c \bar{u} d / s$ whose nonzero entries have been already shown in Eq. (19). The effective Hamiltonian is derived as

$$
\begin{aligned}
\mathcal{H}_{e f f}= & d_{1} \Omega_{b b b}^{-} \bar{B}^{i} \bar{B}^{j}\left(\bar{T}_{c \overline{3}}\right)_{[i k]}\left(H_{8}\right)_{j}^{k} \\
& +d_{2} \Omega_{b b b}^{-} \bar{B}^{i} \bar{B}^{j}\left(\bar{T}_{c 6}\right)_{[i k]}\left(H_{8}\right)_{j}^{k} .
\end{aligned}
$$

The Feynman diagrams for these decays channels are given in Fig. 5 and the decay amplitudes are collected in Table 6, the relations of different decay channels are presented below:

$$
\begin{aligned}
& \Gamma\left(\Omega_{b b b}^{-} \rightarrow B^{-} B^{-} \Lambda_{c}^{+}\right)=2 \Gamma\left(\Omega_{b b b}^{-} \rightarrow B^{-} \bar{B}_{s}^{0} \Xi_{c}^{0}\right), \\
& \Gamma\left(\Omega_{b b b}^{-} \rightarrow B^{-} B^{-} \Xi_{c}^{+}\right)=2 \Gamma\left(\Omega_{b b b}^{-} \rightarrow B^{-} \bar{B}^{0} \Xi_{c}^{0}\right), \\
& \Gamma\left(\Omega_{b b b}^{-} \rightarrow B^{-} B^{-} \Sigma_{c}^{+}\right)=2 \Gamma\left(\Omega_{b b b}^{-} \rightarrow \bar{B}_{s}^{0} B^{-} \Xi_{c}^{\prime 0}\right) \\
& =\Gamma\left(\Omega_{b b b}^{-} \rightarrow B^{-} \bar{B}^{0} \Sigma_{c}^{0}\right), \\
& \Gamma\left(\Omega_{b b b}^{-} \rightarrow B^{-} B^{-} \Xi_{c}^{\prime+}\right)=2 \Gamma\left(\Omega_{b b b}^{-} \rightarrow B^{-} \bar{B}^{0} \Xi_{c}^{\prime 0}\right) \\
& =\Gamma\left(\Omega_{b b b}^{-} \rightarrow B^{-} \bar{B}_{s}^{0} \Omega_{c}^{0}\right) .
\end{aligned}
$$

For the last kind of quark-level transition $b \rightarrow q \bar{q} q$, we will study the decay modes $\Omega_{b b b}^{-} \rightarrow B B T_{8 / 10}$. The charmless $b \rightarrow q / s$ transition is depicted by the weak Hamiltonian $\mathcal{H}_{\text {e.w. }}$,

$\mathcal{H}_{\text {e.w. }}=\frac{G_{F}}{\sqrt{2}}\left\{V_{u b} V_{u q}^{*}\left[C_{1} O_{1}^{\bar{u} u}+C_{2} O_{2}^{\bar{u} u}\right]\right.$ 
Table 5 Amplitudes for $\Omega_{b b b}^{-}$ decays into a $T_{b c}$ and a $B$-meson and a light meson

\begin{tabular}{llll}
\hline Channel & Amplitude & Channel & Amplitude \\
\hline$\Omega_{b b b}^{-} \rightarrow \Xi_{b c}^{0} B^{-}$ & $c_{1} V_{c b} V_{u d}^{*}$ & $\Omega_{b b b}^{-} \rightarrow \Omega_{b c}^{0} B^{-}$ & $c_{1} V_{c b} V_{u s}^{*}$ \\
$\Omega_{b b b}^{-} \rightarrow \Xi_{b c}^{+} B^{-} \pi^{-}$ & $\left(c_{2}+c_{3}\right) V_{c b} V_{u d}^{*}$ & $\Omega_{b b b}^{-} \rightarrow \Xi_{b c}^{+} B^{-} K^{-}$ & $\left(c_{2}+c_{3}\right) V_{c b} V_{u s}^{*}$ \\
$\Omega_{b b b}^{-} \rightarrow \Xi_{b c}^{0} B^{-} \pi^{0}$ & $-\frac{\left(c_{3}-c_{4}\right) V_{c b} V_{u d}^{*}}{\sqrt{2}}$ & $\Omega_{b b b}^{-} \rightarrow \Xi_{b c}^{0} B^{-} \bar{K}^{0}$ & $c_{3} V_{c b} V_{u s}^{*}$ \\
$\Omega_{b b b}^{-} \rightarrow \Xi_{b c}^{0} B^{-} \eta$ & $\frac{\left(c_{3}+c_{4}\right) V_{c b} V_{u d}^{*}}{\sqrt{6}}$ & $\Omega_{b b b}^{-} \rightarrow \Xi_{b c}^{0} \bar{B}^{0} \pi^{-}$ & $\left(c_{2}+c_{4}\right) V_{c b} V_{u d}^{*}$ \\
$\Omega_{b b b}^{-} \rightarrow \Xi_{b c}^{0} \bar{B}^{0} K^{-}$ & $c_{2} V_{c b} V_{u s}^{*}$ & $\Omega_{b b b}^{-} \rightarrow \Xi_{b c}^{0} \bar{B}_{s}^{0} K^{-}$ & $c_{4} V_{c b} V_{u d}^{*}$ \\
$\Omega_{b b b}^{-} \rightarrow \Omega_{b c}^{0} B^{-} \pi^{0}$ & $\frac{c_{4} V_{c b} V_{u s}^{*}}{\sqrt{2}}$ & $\Omega_{b b b}^{-} \rightarrow \Omega_{b c}^{0} B^{-} K^{0}$ & $c_{3} V_{c b} V_{u d}^{*}$ \\
$\Omega_{b b b}^{-} \rightarrow \Omega_{b c}^{0} B^{-} \eta$ & $\frac{\left(c_{4}-2 c_{3}\right) V_{c b} V_{u s}^{*}}{\sqrt{6}}$ & $\Omega_{b b b}^{-} \rightarrow \Omega_{b c}^{0} \bar{B}^{0} \pi^{-}$ & $c_{4} V_{c b} V_{u s}^{*}$ \\
$\Omega_{b b b}^{-} \rightarrow \Omega_{b c}^{0} \bar{B}_{s}^{0} \pi^{-}$ & $c_{2} V_{c b} V_{u d}^{*}$ & $\Omega_{b b b}^{-} \rightarrow \Omega_{b c}^{0} \bar{B}_{s}^{0} K^{-}$ & $\left(c_{2}+c_{4}\right) V_{c b} V_{u s}^{*}$ \\
\hline
\end{tabular}
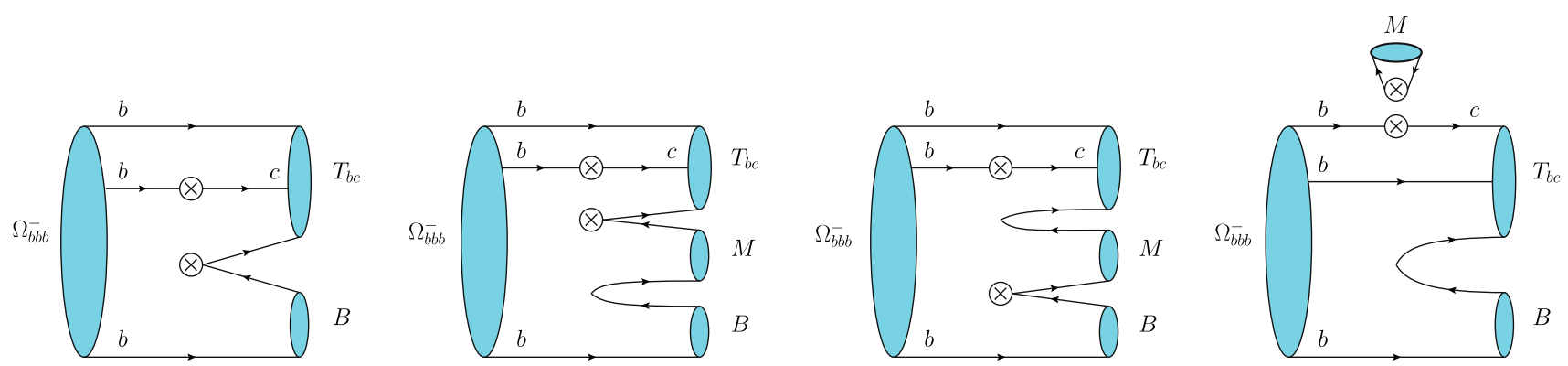

Fig. 4 Feynman diagrams for $\Omega_{b b b}^{-}$decays into a doubly heavy baryon $T_{b c}$, plus a $B$-meson and a light meson

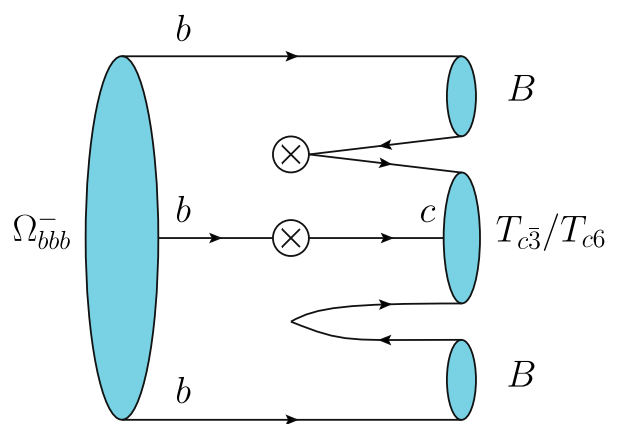

Fig. 5 Feynman diagrams for $\Omega_{b b b}^{-}$decays into two $B$-mesons and a singly charmed baryon (antitriplet or sextet)

$$
\left.-V_{t b} V_{t q}^{*}\left[\sum_{i=3}^{10} C_{i} O_{i}\right]\right\}+ \text { H.c. }
$$

where the explicit expressions for $O_{i}$ can be found in Ref. [58]. Penguin operators in Eq. (24) are not suppressed, they behave as 3 representation in the $\mathrm{SU}(3)$ group,

$$
\begin{aligned}
& \left(H_{3}\right)^{2}=1 \text { (for } \Delta S=0, \text { i.e., } b \rightarrow d \text { case) }, \\
& \left(H_{3}\right)^{3}=1 \text { (for } \Delta S=1, \text { i.e., } b \rightarrow s \text { case) } .
\end{aligned}
$$

Tree operators can be decomposed in terms of a vector $H_{3}$, two traceless tensors $H_{\overline{6}}$ and $H_{15}$ whose nonzero entries are

$$
\left\{\begin{array}{l}
\left(H_{\overline{6}}\right)_{1}^{12}=-\left(H_{\overline{6}}\right)_{1}^{21}=\left(H_{\overline{6}}\right)_{3}^{23}=-\left(H_{\overline{6}}\right)_{3}^{32}=1 \\
2\left(H_{15}\right)_{1}^{12}=2\left(H_{15}\right)_{1}^{21}=-3\left(H_{15}\right)_{2}^{22} \\
=-6\left(H_{15}\right)_{3}^{23}=-6\left(H_{15}\right)_{3}^{32}=6 . \\
\quad(\text { for } \Delta S=0, \text { i.e. }, b \rightarrow d \text { case })
\end{array}\right.
$$

Table 6 Amplitudes for $\Omega_{b b b}^{-}$ decays into two $B$-mesons and a singly charmed baryon (antitriplet or sextet)

\begin{tabular}{llll}
\hline Channel & Amplitude & Channel & Amplitude \\
\hline$\Omega_{b b b}^{-} \rightarrow B^{-} B^{-} \Lambda_{c}^{+}$ & $2 d_{1} V_{c b} V_{u d}^{*}$ & $\Omega_{b b b}^{-} \rightarrow B^{-} B^{-} \Xi_{c}^{+}$ & $2 d_{1} V_{c b} V_{u s}^{*}$ \\
$\Omega_{b b b}^{-} \rightarrow B^{-} \bar{B}^{0} \Xi_{c}^{0}$ & $d_{1} V_{c b} V_{u s}^{*}$ & $\Omega_{b b b}^{-} \rightarrow B^{-} \bar{B}_{s}^{0} \Xi_{c}^{0}$ & $-d_{1} V_{c b} V_{u d}^{*}$ \\
$\Omega_{b b b}^{-} \rightarrow B^{-} B^{-} \Sigma_{c}^{+}$ & $\sqrt{2} d_{2} V_{c b} V_{u d}^{*}$ & $\Omega_{b b b}^{-} \rightarrow B^{-} B^{-} \Xi_{c}^{\prime+}$ & $\sqrt{2} d_{2} V_{c b} V_{u s}^{*}$ \\
$\Omega_{b b b}^{-} \rightarrow B^{-} \bar{B}^{0} \Sigma_{c}^{0}$ & $d_{2} V_{c b} V_{u d}^{*}$ & $\Omega_{b b b}^{-} \rightarrow B^{-} \bar{B}^{0} \Xi_{c}^{\prime 0}$ & $\frac{d_{2} V_{c b} V_{u s}^{*}}{\sqrt{2}}$ \\
$\Omega_{b b b}^{-} \rightarrow B^{-} \bar{B}_{s}^{0} \Xi_{c}^{\prime 0}$ & $\frac{d_{2} V_{c b} V_{u d}^{*}}{\sqrt{2}}$ & $\Omega_{b b b}^{-} \rightarrow B^{-} \bar{B}_{s}^{0} \Omega_{c}^{0}$ & $d_{2} V_{c b} V_{u s}^{*}$ \\
\hline
\end{tabular}


Table 7 Amplitudes for $\Omega_{b b b}^{-}$decays into two $B$-mesons and a light baryon (octet)

\begin{tabular}{llll}
\hline Channel & Amplitude & Channel & Amplitude \\
\hline$\Omega_{b b b}^{-} \rightarrow B^{-} B^{-} p$ & $2\left(f_{2}-f_{3}+2 f_{4}+3 f_{5}\right)$ & $\Omega_{b b b}^{-} \rightarrow B^{-} \bar{B}^{0}{ }_{n}$ & $f_{2}-f_{3}+2 f_{4}-5 f_{5}$ \\
$\Omega_{b b b}^{-} \rightarrow B^{-} \bar{B}_{s}^{0} \Lambda^{0}$ & $\frac{-3 f_{2}+f_{3}-2 f_{4}+3 f_{5}}{\sqrt{6}}$ & $\Omega_{b b b}^{-} \rightarrow B^{-} \bar{B}_{s}^{0} \Sigma^{0}$ & $-\frac{f_{2}+f_{3}-2 f_{4}+7 f_{5}}{\sqrt{2}}$ \\
$\Omega_{b b b}^{-} \rightarrow \bar{B}^{0} \bar{B}_{s}^{0} \Sigma^{-}$ & $-f_{2}-f_{3}+2 f_{4}+f_{5}$ & $\Omega_{b b b}^{-} \rightarrow \bar{B}_{s}^{0} \bar{B}_{s}^{0} \Xi^{-}$ & $2\left(-f_{2}-f_{3}+2 f_{4}+f_{5}\right)$ \\
$\Omega_{b b b}^{-} \rightarrow B^{-} B^{-} \Sigma^{+}$ & $2\left(-f_{2}^{\prime}+f_{3}^{\prime}-2 f_{4}^{\prime}-3 f_{5}^{\prime}\right)$ & $\Omega_{b b b}^{-} \rightarrow B^{-} \bar{B}^{0} \Lambda^{0}$ & $\sqrt{\frac{2}{3}}\left(f_{3}^{\prime}-2 f_{4}^{\prime}+6 f_{5}^{\prime}\right)$ \\
$\Omega_{b b b}^{-} \rightarrow B^{-} \bar{B}^{0} \Sigma^{0}$ & $\sqrt{2}\left(f_{2}^{\prime}+f_{5}^{\prime}\right)$ & $\Omega_{b b b}^{-} \rightarrow B^{-} \bar{B}_{s}^{0} \Xi^{0}$ & $-f_{2}^{\prime}+f_{3}^{\prime}-2 f_{4}^{\prime}+5 f_{5}^{\prime}$ \\
$\Omega_{b b b}^{-} \rightarrow \bar{B}^{0} \bar{B}^{0} \Sigma^{-}$ & $2\left(f_{2}^{\prime}+f_{3}^{\prime}-2 f_{4}^{\prime}-f_{5}^{\prime}\right)$ & $\Omega_{b b b}^{-} \rightarrow \bar{B}_{s}^{0} \bar{B}^{0} \Xi^{-}$ & $f_{2}^{\prime}+f_{3}^{\prime}-2 f_{4}^{\prime}-f_{5}^{\prime}$ \\
\hline
\end{tabular}

Fig. 6 Feynman diagrams for $\Omega_{b b b}^{-}$decays into two $B$-mesons and a light baryon (octet or decuplet)
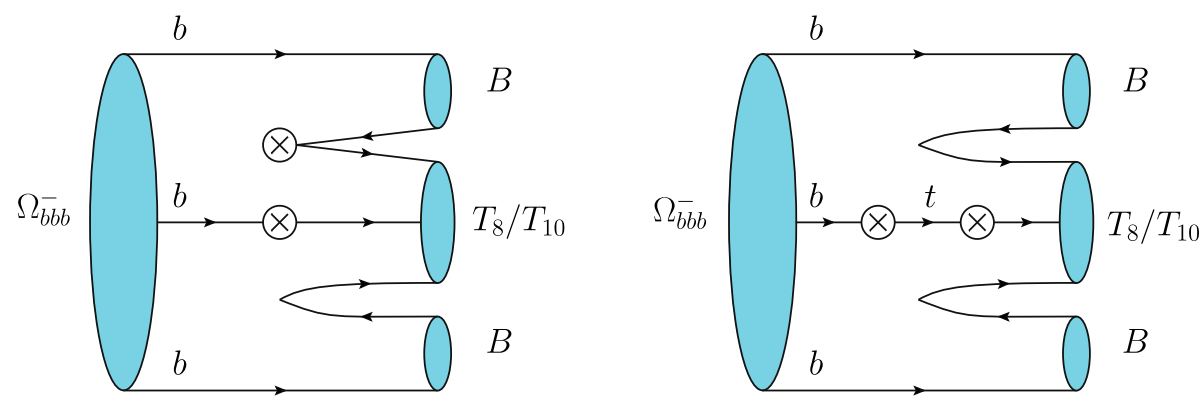

$\left\{\begin{array}{l}\left(H_{\overline{6}}\right)_{1}^{13}=-\left(H_{\overline{6}}\right)_{1}^{31}=\left(H_{\overline{6}}\right)_{2}^{32}=-\left(H_{\overline{6}}\right)_{2}^{23}=1, \\ 2\left(H_{15}\right)_{1}^{13}=2\left(H_{15}\right)_{1}^{31}=-3\left(H_{15}\right)_{3}^{33} \\ =-6\left(H_{15}\right)_{2}^{32}=-6\left(H_{15}\right)_{2}^{23}=6 .\end{array}\right.$

$$
\text { (for } \Delta S=1 \text {, i.e., } b \rightarrow s \text { case) }
$$

Thus, for $\Omega_{b b b}^{-}$decays into two $B$-mesons and one light baryon (octet), the effective Hamiltonian is given as

$$
\begin{aligned}
\mathcal{H}_{e f f}= & f_{1} \Omega_{b b b}^{-} \bar{B}^{i} \bar{B}^{j} \varepsilon_{i j k}\left(\bar{T}_{8}\right)_{l}^{k}\left(H_{3}\right)^{l} \\
& +f_{2} \Omega_{b b b}^{-} \bar{B}^{i} \bar{B}^{l} \varepsilon_{i j k}\left(\bar{T}_{8}\right)_{l}^{k}\left(H_{3}\right)^{j} \\
& +f_{3} \Omega_{b b b}^{-} \bar{B}^{i} \bar{B}^{m} \varepsilon_{i j k}\left(\bar{T}_{8}\right)_{l}^{k}\left(H_{\overline{6}}\right)_{m}^{j l} \\
& +f_{4} \Omega_{b b b}^{-} \bar{B}^{m} \bar{B}^{l} \varepsilon_{i j k}\left(\bar{T}_{8}\right)_{l}^{k}\left(H_{\overline{6}}\right)_{m}^{i j} \\
& +f_{5} \Omega_{b b b}^{-} \bar{B}^{i} \bar{B}^{m} \varepsilon_{i j k}\left(\bar{T}_{8}\right)_{l}^{k}\left(H_{15}\right)_{m}^{j l} .
\end{aligned}
$$

Similarly, the decay amplitudes are obtained and collected in Table 7, the corresponding Feynman diagrams are presented in Fig. 6. Two relations for decay widths can be read off

$$
\begin{gathered}
\Gamma\left(\Omega_{b b b}^{-} \rightarrow \bar{B}^{0} \bar{B}_{s}^{0} \Sigma^{-}\right)=\frac{1}{2} \Gamma\left(\Omega_{b b b}^{-} \rightarrow \bar{B}_{s}^{0} \bar{B}_{s}^{0} \Xi^{-}\right), \\
\Gamma\left(\Omega_{b b b}^{-} \rightarrow \bar{B}^{0} \bar{B}^{0} \Sigma^{-}\right)=2 \Gamma\left(\Omega_{b b b}^{-} \rightarrow \bar{B}^{0} \bar{B}_{s}^{0} \Xi^{-}\right) .
\end{gathered}
$$

For the case $\Omega_{b b b}^{-}$decays into two $B$-mesons and one light baryon (decuplet), the effective Hamiltonian is given as

$$
\begin{aligned}
& \mathcal{H}_{e f f}=g_{1} \Omega_{b b b}^{-} \bar{B}^{i} \bar{B}^{j}\left(\bar{T}_{10}\right)_{i j k}\left(H_{3}\right)^{k} \\
& +g_{2} \Omega_{b b b}^{-} \bar{B}^{i} \bar{B}^{l}\left(\bar{T}_{10}\right)_{i j k}\left(H_{15}\right)_{l}^{j k} .
\end{aligned}
$$

The decay amplitudes are obtained and collected in Table 8 . Various relations for decay widths can be deduced:

$$
\begin{aligned}
& \Gamma\left(\Omega_{b b b}^{-} \rightarrow B^{-} \bar{B}^{0} \Delta^{0}\right)=2 \Gamma\left(\Omega_{b b b}^{-} \rightarrow B^{-} \bar{B}_{s}^{0} \Sigma^{\prime 0}\right), \\
& \Gamma\left(\Omega_{b b b}^{-} \rightarrow \bar{B}^{0} \bar{B}_{s}^{0} \Sigma^{\prime-}\right)=2 \Gamma\left(\Omega_{b b b}^{-} \rightarrow \bar{B}_{s}^{0} \bar{B}_{s}^{0} \Xi^{\prime-}\right), \\
& \Gamma\left(\Omega_{b b b}^{-} \rightarrow \bar{B}^{0} B^{-} \Sigma^{\prime 0}\right)=\frac{1}{2} \Gamma\left(\Omega_{b b b}^{-} \rightarrow \bar{B}_{s}^{0} B^{-} \Xi^{\prime 0}\right), \\
& \Gamma\left(\Omega_{b b b}^{-} \rightarrow \bar{B}^{0} \bar{B}_{s}^{0} \Xi^{\prime-}\right)=\frac{2}{3} \Gamma\left(\Omega_{b b b}^{-} \rightarrow \bar{B}_{s}^{0} \bar{B}_{s}^{0} \Omega^{-}\right), \\
& \Gamma\left(\Omega_{b b b}^{-} \rightarrow \bar{B}^{0} \bar{B}^{0} \Delta^{-}\right)=\frac{3}{2} \Gamma\left(\Omega_{b b b}^{-} \rightarrow \bar{B}_{s}^{0} \bar{B}^{0} \Sigma^{\prime-}\right) \\
& =3 \Gamma\left(\Omega_{b b b}^{-} \rightarrow \bar{B}_{s}^{0} \bar{B}_{s}^{0} \Xi^{\prime-}\right), \\
& \Gamma\left(\Omega_{b b b}^{-} \rightarrow \bar{B}^{0} \bar{B}^{0} \Sigma^{\prime-}\right)=\frac{1}{2} \Gamma\left(\Omega_{b b b}^{-} \rightarrow \bar{B}_{s}^{0} \bar{B}^{0} \Xi^{\prime-}\right) \\
& =\frac{1}{3} \Gamma\left(\Omega_{b b b}^{-} \rightarrow \bar{B}_{s}^{0} \bar{B}_{s}^{0} \Omega^{-}\right) .
\end{aligned}
$$

A few remarks are given in order:

1. The channels in Tables 7 and 8 are arranged according to its quark level transition is whether $b \rightarrow d$ or $b \rightarrow s$. Note that their CKM matrix elements which have been absorbed in the nonperturbative coefficients are different, therefore the coefficients in $\mathrm{SU}(3)$ irreducible amplitudes for the $b \rightarrow s$ transition are primed $\left(f_{i}^{\prime}\right.$ and $g_{i}^{\prime}$ ).

2. One can infer that the typical branching fractions are at the order $10^{-6}$ through a simple analogy with the $B$ meson decay data. Thus there is little chance to discover the triply bottom baryon through these decay channels, but they can be utilized to study the direct CP asymmetries once large amount of data have been accumulated in future [46]. 
Table 8 Amplitudes for $\Omega_{b b b}^{-}$ decays into two $B$-mesons and a light baryon (decuplet)

Fig. 7 Feynman diagrams for $\Omega_{c b b}^{0}$ decays into a doubly heavy baryon $T_{b c}$ and a $D$-meson. The final hadron into which the spectator $c$ quark flows is marked with red

Table 9 Cabibbo-allowed decay channels for $\Omega_{c c c}^{++}$and CKM-allowed decay channels for $\Omega_{b b b}^{-}$

\begin{tabular}{llll}
\hline Channel & Amplitude & Channel & Amplitude \\
\hline$\Omega_{b b b}^{-} \rightarrow B^{-} B^{-} \Delta^{+}$ & $\sqrt{\frac{4}{3}}\left(g_{1}+6 g_{2}\right)$ & $\Omega_{b b b}^{-} \rightarrow B^{-} \bar{B}^{0} \Delta^{0}$ & $\sqrt{\frac{4}{3}}\left(g_{1}+2 g_{2}\right)$ \\
$\Omega_{b b b}^{-} \rightarrow B^{-} \bar{B}_{s}^{0} \Sigma^{\prime 0}$ & $\sqrt{\frac{2}{3}}\left(g_{1}+2 g_{2}\right)$ & $\Omega_{b b b}^{-} \rightarrow \bar{B}^{0} \bar{B}^{0} \Delta^{-}$ & $2\left(g_{1}-2 g_{2}\right)$ \\
$\Omega_{b b b}^{-} \rightarrow \bar{B}^{0} \bar{B}_{s}^{0} \Sigma^{\prime-}$ & $\sqrt{\frac{4}{3}}\left(g_{1}-2 g_{2}\right)$ & $\Omega_{b b b}^{-} \rightarrow \bar{B}_{s}^{0} \bar{B}_{s}^{0} \Xi^{\prime-}$ & $\sqrt{\frac{4}{3}}\left(g_{1}-2 g_{2}\right)$ \\
$\Omega_{b b b}^{-} \rightarrow B^{-} B^{-} \Sigma^{\prime+}$ & $\sqrt{\frac{4}{3}}\left(g_{1}^{\prime}+6 g_{2}^{\prime}\right)$ & $\Omega_{b b b}^{-} \rightarrow B^{-} \bar{B}^{0} \Sigma^{\prime 0}$ & $\sqrt{\frac{2}{3}}\left(g_{1}^{\prime}+2 g_{2}^{\prime}\right)$ \\
$\Omega_{b b b}^{-} \rightarrow B^{-} \bar{B}_{s}^{0} \Xi^{\prime 0}$ & $\sqrt{\frac{4}{3}}\left(g_{1}^{\prime}+2 g_{2}^{\prime}\right)$ & $\Omega_{b b b}^{-} \rightarrow \bar{B}^{0} \bar{B}^{0} \Sigma^{\prime-}$ & $\sqrt{\frac{4}{3}}\left(g_{1}^{\prime}-2 g_{2}^{\prime}\right)$ \\
$\Omega_{b b b}^{-} \rightarrow \bar{B}^{0} \bar{B}_{s}^{0} \Xi^{\prime-}$ & $\sqrt{\frac{4}{3}}\left(g_{1}^{\prime}-2 g_{2}^{\prime}\right)$ & $\Omega_{b b b}^{-} \rightarrow \bar{B}_{s}^{0} B^{-} \Xi^{\prime 0}$ & $\sqrt{\frac{4}{3}}\left(g_{1}^{\prime}+2 g_{2}^{\prime}\right)$ \\
$\Omega_{b b b}^{-} \rightarrow \bar{B}_{s}^{0} \bar{B}_{s}^{0} \Omega^{-}$ & $2\left(g_{1}^{\prime}-2 g_{2}^{\prime}\right)$ & & \\
\hline
\end{tabular}
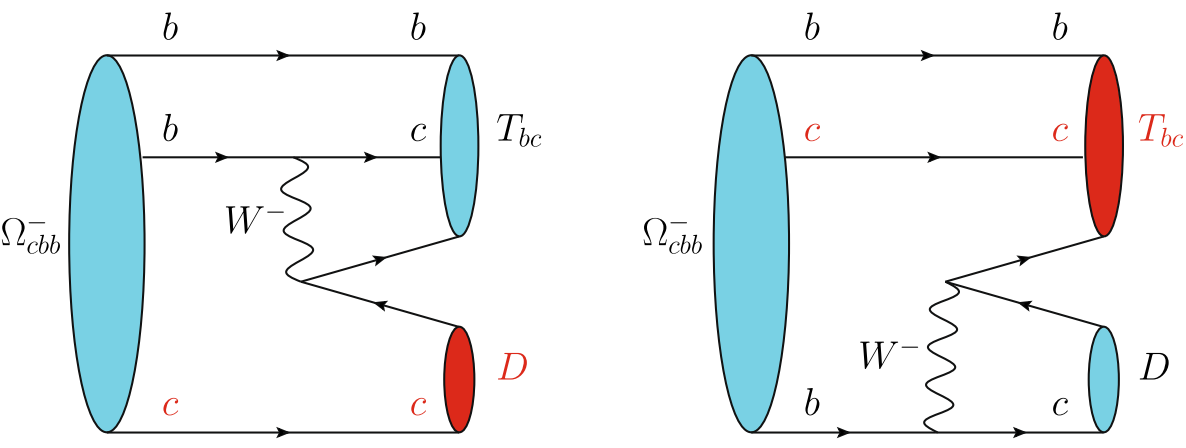

\begin{tabular}{llll}
\hline Channel & Channel & Channel & Channel \\
\hline$\Omega_{c c c}^{++} \rightarrow D^{0} D^{+} \Sigma^{+}$ & $\Omega_{c c c}^{++} \rightarrow D^{+} D^{+} \Lambda^{0}$ & $\Omega_{c c c}^{++} \rightarrow D^{+} D^{+} \Sigma^{0}$ & $\Omega_{c c c}^{++} \rightarrow D^{+} D_{s}^{+} \Xi^{0}$ \\
$\Omega_{c c c}^{++} \rightarrow D^{0} D^{+} \Sigma^{\prime+}$ & $\Omega_{c c c}^{++} \rightarrow D^{+} D^{+} \Sigma^{\prime 0}$ & $\Omega_{c c c}^{++} \rightarrow D_{s}^{+} D^{+} \Xi^{\prime 0}$ & \\
$\Omega_{b b b}^{-} \rightarrow \Xi_{b c}^{0} B_{c}$ & $\Omega_{b b b}^{-} \rightarrow \Xi_{b c}^{0} B^{-}$ & & \\
$\Omega_{b b b}^{-} \rightarrow \Omega_{b c}^{0} K^{0} B_{c}$ & $\Omega_{b b b}^{-} \rightarrow \Xi_{b c}^{+} \pi^{-} B_{c}$ & $\Omega_{b b b}^{-} \rightarrow \Xi_{b c}^{0} \pi^{0} B_{c}$ & $\Omega_{b b b}^{-} \rightarrow \Xi_{b c}^{0} \eta B_{c}$ \\
$\Omega_{b b b}^{-} \rightarrow \Omega_{b c}^{0} \bar{B}_{s}^{0} \pi^{-}$ & $\Omega_{b b b}^{-} \rightarrow \Xi_{b c}^{+} B^{-} \pi^{-}$ & $\Omega_{b b b}^{-} \rightarrow \Xi_{b c}^{0} B^{-} \pi^{0}$ & $\Omega_{b b b}^{-} \rightarrow \Xi_{b c}^{0} B^{-} \eta$ \\
$\Omega_{b b b}^{-} \rightarrow \Xi_{b c}^{0} \bar{B}^{0} \pi^{-}$ & $\Omega_{b b b}^{-} \rightarrow \Xi_{b c}^{0} \bar{B}_{s}^{0} K^{-}$ & $\Omega_{b b b}^{-} \rightarrow \Omega_{b c}^{0} B^{-} K^{0}$ & $\Omega_{b b b}^{-} \rightarrow B^{-} B^{-} \Lambda_{c}^{+}$ \\
$\Omega_{b b b}^{-} \rightarrow B^{-} \bar{B}_{s}^{0} \Xi_{c}^{0}$ & $\Omega_{b b b}^{-} \rightarrow B^{-} B^{-} \Sigma_{c}^{+}$ & $\Omega_{b b b}^{-} \rightarrow B^{-} \bar{B}^{0} \Sigma_{c}^{0}$ & $\Omega_{b b b}^{-} \rightarrow B^{-} \bar{B}_{s}^{0} \Xi_{c}^{\prime 0}$ \\
\hline
\end{tabular}

\subsection{Nonleptonic $\Omega_{c c b}^{+}$and $\Omega_{c b b}^{0}$ decays}

Most decay modes of $\Omega_{c c b}^{+}$and $\Omega_{c b b}^{0}$ can be obtained from the results of $\Omega_{c c c}^{++}$and $\Omega_{b b b}^{-}$with some replacements. For example, decays of $\Omega_{c c b}^{+}$induced by the charm quark can be obtained from the ones of $\Omega_{c c c}^{++}$through replacing one charmed meson by the corresponding bottom meson, a charmed baryon by the corresponding bottom baryon, or a doubly charmed baryon $T_{c c}$ by its counterpart $T_{b c}$. In addition, there is another kind of decay modes of the mixed triply heavy baryons, i.e., the $W$-exchange transition which has been discussed in Ref. [46]. Therefore we won't explicitly show the effective Hamiltonian for various decay modes of $\Omega_{c c b}^{+}$and $\Omega_{c b b}^{0}$ here.

As we have mentioned before, one significant advantage of the $\mathrm{SU}(3)$ analysis is that it is independent of the fac- torization details, this can be clearly revealed through, for instance, the weak decay of $\Omega_{c b b}^{0}$ into a mixed doubly heavy baryon $T_{b c}$ and a $D$-meson. There are two possibilities in this weak decay: the spectator $c$ quark in $\Omega_{c b b}^{0}$ may flow in the final mixed doubly heavy baryon $T_{b c}$ or interact with a light antiquark to form a $D$-meson after hadronization. The typical Feynman diagrams corresponding to these two cases are depicted in Fig. 7. However, from the perspective of flavor SU(3) analysis, there is no difference between these two cases since the heavy $c$ quark is $\mathrm{SU}(3)$ singlet. As long as one assumes the flavor SU(3) symmetry is approximately preserved in the weak decays of triply heavy baryon, the SU(3) transformation invariant effective Hamiltonian can be written as $\mathcal{H}_{\text {eff }}=h_{1} \Omega_{c b b}^{0}\left(\bar{T}_{b c}\right)_{i} \bar{D}^{j}\left(H_{8}\right)_{j}^{i}$. 


\section{Discussions and conclusions}

Based on the above analysis in Sect. 3, a collection of Cabibbo-allowed decay channels for $\Omega_{c c c}^{++}$and CKM-allowed decay channels for $\Omega_{b b b}^{-}$has been presented in Table 9. For the $\Omega_{c c c}^{++}$decay, the branching fractions for the Cabibboallowed processes might reach a few percent, thus presumably lead to discovery of triply charmed baryon. For the $\Omega_{b b b}^{-}$ decay, the CKM-allowed largest branching fraction might reach $10^{-3}$, which would be even much smaller when considering detecting charmless final states in experiment.

The triply heavy baryons are of considerable theoretical interests, since they are free of light quark contamination and can help to probe the interplay between perturbative and nonperturbative QCD $[59,60]$. The observation of doubly heavy baryon makes it more reliable to look forward the triply heavy baryon in future colliders such as the high luminosity LHC.

This work is an extension of previous studies, we have systematically analyzed the nonleptonic weak decays of triply heavy baryons. Decay amplitudes for these processes have been parametrized in terms of the $\mathrm{SU}(3)$ irreducible nonperturbative amplitudes $\left(a_{i}\right.$ 's $\sim g_{i}$ 's). A number of relations for the partial decay widths can be deduced from these results and can be examined once we have a large amount of data in future. We also list the decay channels of triply heavy baryons and some cascade decay modes of $\Omega_{c c c}^{++}$which likely to be used to reconstructing in experiments. It is worth emphasizing here, that the triply heavy baryons are still absent in particle data booklet after the discovery of the heavy quarkonium $J / \psi$ over four decades. Therefore we encourage our experimental colleagues performing searches of this kind of particles since the reward could be high and will be a milestone in hadron physics.

Acknowledgements The authors are grateful to Professor Wei Wang and Dr. Ye Xing for inspiring discussions and valuable comments. When this work was about to finalize, Zhengzhou encountered unprecedented rainstorm, we want to express our gratitude to the people of Zhengzhou for their bravery in this extreme weather event. F.H is supported in part by National Natural Science Foundation of China under Grant nos. 11735010, U2032102, 11653003, Natural Science Foundation of Shanghai under Grant no. 15DZ2272100. J.X. is supported in part by National Natural Science Foundation of China under Grant no. 12047545 and 12105247.

Data Availability Statement This manuscript has no associated data or the data will not be deposited. [Authors' comment: The reason why the data will not be deposited is there are no external data associated with the manuscript.]

Open Access This article is licensed under a Creative Commons Attribution 4.0 International License, which permits use, sharing, adaptation, distribution and reproduction in any medium or format, as long as you give appropriate credit to the original author(s) and the source, provide a link to the Creative Commons licence, and indicate if changes were made. The images or other third party material in this article are included in the article's Creative Commons licence, unless indicated otherwise in a credit line to the material. If material is not included in the article's Creative Commons licence and your intended use is not permitted by statutory regulation or exceeds the permitted use, you will need to obtain permission directly from the copyright holder. To view a copy of this licence, visit http://creativecomm ons.org/licenses/by/4.0/.

Funded by $\mathrm{SCOAP}^{3}$.

\section{References}

1. J. Vijande, H. Garcilazo, A. Valcarce, F. Fernandez, Phys. Rev. D 70, 054022 (2004). https://doi.org/10.1103/PhysRevD.70.054022. arXiv:hep-ph/0408274

2. N. Brambilla, A. Vairo, T. Rosch, Phys. Rev. D 72, 034021 (2005). https://doi.org/10.1103/PhysRevD.72.034021. arXiv:hep-ph/0506065

3. J.R. Zhang, M.Q. Huang, Phys. Lett. B 674, 28-35 (2009). https://doi.org/10.1016/j.physletb.2009.02.056. arXiv:0902.3297 [hep-ph]

4. C. Patrignani et al. [Particle Data Group], Chin. Phys. C 40(10), 100001 (2016). https://doi.org/10.1088/1674-1137/40/10/100001

5. M. Ablikim et al. [BESIII], Phys. Rev. Lett. 116(5), 052001 (2016). https://doi.org/10.1103/PhysRevLett.116.052001. arXiv:1511.08380 [hep-ex]

6. R. Aaij et al. [LHCb], Phys. Rev. Lett. 119(11), 112001 (2017). $\quad$ https://doi.org/10.1103/PhysRevLett.119.112001. arXiv:1707.01621 [hep-ex]

7. R. Aaij et al. [LHCb], Phys. Rev. Lett. 121(16), 162002 (2018). https://doi.org/10.1103/PhysRevLett.121.162002. arXiv:1807.01919 [hep-ex]

8. S.P. Baranov, V.L. Slad, Phys. Atom. Nucl. 67, 808-814 (2004). https://doi.org/10.1134/1.1707141. arXiv:hep-ph/0603090

9. Y.Q. Chen, S.Z. Wu, JHEP 08, 144 (2011). https://doi.org/10.1007/ JHEP08(2011)144. arXiv:1106.0193 [hep-ph] (erratum: JHEP 09, 089 (2011))

10. Y. Jia, JHEP 10, 073 (2006). https://doi.org/10.1088/1126-6708/ 2006/10/073. arXiv:hep-ph/0607290

11. S. Meinel, Phys. Rev. D 82, 114514 (2010). https://doi.org/10. 1103/PhysRevD.82.114514. arXiv:1008.3154 [hep-lat]

12. Z.G. Wang, Commun. Theor. Phys. 58, 723-731 (2012). https:// doi.org/10.1088/0253-6102/58/5/17. arXiv:1112.2274 [hep-ph]

13. T.M. Aliev, K. Azizi, M. Savci, JHEP 04, 042 (2013). https://doi. org/10.1007/JHEP04(2013)042. arXiv:1212.6065 [hep-ph]

14. Z.S. Brown, W. Detmold, S. Meinel, K. Orginos, Phys. Rev. D 90(9), 094507 (2014). https://doi.org/10.1103/PhysRevD.90. 094507. arXiv:1409.0497 [hep-lat]

15. K.W. Wei, B. Chen, X.H. Guo, Phys. Rev. D 92(7), 076008 (2015). https://doi.org/10.1103/PhysRevD.92.076008. arXiv:1503.05184 [hep-ph]

16. K. Thakkar, A. Majethiya, P.C. Vinodkumar, Eur. Phys. J. Plus 131(9), 339 (2016). https://doi.org/10.1140/epjp/i2016-16339-4. arXiv: 1609.05444 [hep-ph]

17. M.A. GomshiNobary, R. Sepahvand, Phys. Rev. D 71, 034024 (2005). https://doi.org/10.1103/PhysRevD.71.034024. arXiv:hep-ph/0406148

18. J.M. Flynn, E. Hernandez, J. Nieves, Phys. Rev. D 85, 014012 (2012). https://doi.org/10.1103/PhysRevD.85.014012. arXiv:1110.2962 [hep-ph]

19. C.Q. Geng, Y.K. Hsiao, C.W. Liu, T.H. Tsai, JHEP 11, 147 (2017). https://doi.org/10.1007/JHEP11(2017)147. arXiv:1709.00808 [hep-ph] 
20. M.S. Liu, Q.F. Lü, X.H. Zhong, Phys. Rev. D 101(7), 074031 (2020). https://doi.org/10.1103/PhysRevD.101.074031. arXiv:1912.11805 [hep-ph]

21. G. Yang, J. Ping, P.G. Ortega, J. Segovia, Chin. Phys. C 44(2), 023102 (2020). https://doi.org/10.1088/1674-1137/44/2/023102. arXiv:1904.10166 [hep-ph]

22. M.J. Savage, M.B. Wise, Phys. Rev. D 39, 3346 (1989). https:// doi.org/10.1103/PhysRevD.39.3346. https://doi.org/10.1103/ PhysRevD.40.3127 (Erratum: [Phys. Rev. D 40, 3127 (1989)])

23. M. Gronau, O.F. Hernandez, D. London, J.L. Rosner, Phys. Rev. D 50, 4529 (1994). https://doi.org/10.1103/PhysRevD.50.4529. arXiv:hep-ph/9404283

24. B. Grinstein, R.F. Lebed, Phys. Rev. D 53, 6344 (1996). https:// doi.org/10.1103/PhysRevD.53.6344. arXiv:hep-ph/9602218

25. X.G. He, Eur. Phys. J. C 9, 443 (1999). https://doi.org/10.1007/ s100529900064. arXiv:hep-ph/9810397

26. N.G. Deshpande, X.G. He, J.Q. Shi, Phys. Rev. D (2000). https:// doi.org/10.1103/PhysRevD.62.034018. arXiv:hep-ph/0002260

27. N.G. Deshpande, X.G. He, Phys. Rev. Lett. 75, 1703 (1995). https:// doi.org/10.1103/PhysRevLett.75.1703. arXiv:hep-ph/9412393

28. C.W. Chiang, M. Gronau, Z. Luo, J.L. Rosner, D.A. Suprun, Phys. Rev. D 69, 034001 (2004). https://doi.org/10.1103/PhysRevD.69. 034001. arXiv:hep-ph/0307395

29. C.W. Chiang, Y.F. Zhou, JHEP 0612, 027 (2006). https://doi.org/ 10.1088/1126-6708/2006/12/027. arXiv:hep-ph/0609128

30. Y. Li, C.D. Lü, W. Wang, Phys. Rev. D 77, 054001 (2008). https:// doi.org/10.1103/PhysRevD.77.054001. arXiv:0711.0497 [hep-ph]

31. S.H. Zhou, Y.B. Wei, Q. Qin, Y. Li, F.S. Yu, C.D. Lu, Phys. Rev. D 92(9), 094016 (2015). https://doi.org/10.1103/PhysRevD. 92.094016. arXiv:1509.04060 [hep-ph]

32. H.Y. Jiang, F.S. Yu, Q. Qin, H.N. Li, C.D. Lü, Chin. Phys. C (2018). https://doi.org/10.1088/1674-1137/42/6/063101. arXiv:1705.07335 [hep-ph]

33. W. Wang, Y.M. Wang, D.S. Yang, C.D. Lü, Phys. Rev. D 78, 034011 (2008). https://doi.org/10.1103/PhysRevD.78.034011. arXiv:0801.3123 [hep-ph]

34. C.W. Chiang, Y.F. Zhou, JHEP 0903, 055 (2009). https://doi.org/ 10.1088/1126-6708/2009/03/055. arXiv:0809.0841 [hep-ph]

35. H.Y. Cheng, C.W. Chiang, A.L. Kuo, Phys. Rev. D 91(1), 014011 (2015). https://doi.org/10.1103/PhysRevD.91.014011. arXiv:1409.5026 [hep-ph]

36. X.G. He, G.N. Li, D. Xu, Phys. Rev. D 91(1), 014029 (2015). https://doi.org/10.1103/PhysRevD.91.014029. arXiv:1410.0476 [hep-ph]

37. X.G. He, G.N. Li, Phys. Lett. B 750, 82 (2015). https://doi.org/10. 1016/j.physletb.2015.08.048. arXiv:1501.00646 [hep-ph]

38. M. He, X.G. He, G.N. Li, Phys. Rev. D 92(3), 036010 (2015). https://doi.org/10.1103/PhysRevD.92.036010. arXiv:1507.07990 [hep-ph]

39. C.D. Lü, W. Wang, F.S. Yu, Phys. Rev. D 93(5), 056008 (2016). https://doi.org/10.1103/PhysRevD.93.056008. arXiv:1601.04241 [hep-ph]
40. H.Y. Cheng, C.W. Chiang, A.L. Kuo, Phys. Rev. D 93(11), 114010 (2016). https://doi.org/10.1103/PhysRevD.93.114010. arXiv:1604.03761 [hep-ph]

41. H.Y. Cheng, C.W. Chiang, Phys. Rev. D 86, 014014 (2012). https:// doi.org/10.1103/PhysRevD.86.014014. arXiv:1205.0580 [hep-ph]

42. H.N. Li, C.D. Lü, F.S. Yu, Phys. Rev. D 86, 036012 (2012). https:// doi.org/10.1103/PhysRevD.86.036012. arXiv:1203.3120 [hep-ph]

43. H.N. Li, C.D. Lü, Q. Qin, F.S. Yu, Phys. Rev. D 89(5), 054006 (2014). https://doi.org/10.1103/PhysRevD.89.054006. arXiv:1305.7021 [hep-ph]

44. W. Wang, Z.P. Xing, J. Xu, Eur. Phys. J. C 77(11), 800 (2017). https://doi.org/10.1140/epjc/s10052-017-5363-y. arXiv:1707.06570 [hep-ph]

45. Y.J. Shi, W. Wang, Y. Xing, J. Xu, Eur. Phys. J. C 78(1), 56 (2018). https://doi.org/10.1140/epjc/s10052-018-5532-7. arXiv:1712.03830 [hep-ph]

46. W. Wang, J. Xu, Phys. Rev. D 97(9), 093007 (2018). https://doi. org/10.1103/PhysRevD.97.093007. arXiv:1803.01476 [hep-ph]

47. D.M. Li, X.R. Zhang, Y. Xing, J. Xu, arXiv:2101.12574 [hep-ph]

48. Q.A. Zhang, Eur. Phys. J. C 78(12), 1024 (2018). https://doi.org/ 10.1140/epjc/s10052-018-6481-x. arXiv:1811.02199 [hep-ph]

49. P.A. Zyla et al. [Particle Data Group], PTEP 2020(8), 083C01 (2020). https://doi.org/10.1093/ptep/ptaa104

50. S.H. Zhou, R.H. Li, Z.Y. Wei, C.D. Lu, arXiv:2107.11079 [hep-ph]

51. J.J. Han, H.Y. Jiang, W. Liu, Z.J. Xiao, F.S. Yu, Chin. Phys. C 45(5), 053105 (2021). https://doi.org/10.1088/1674-1137/abec68. arXiv:2101.12019 [hep-ph]

52. N. Sharma, R. Dhir, Phys. Rev. D 96(11), 113006 (2017). https:// doi.org/10.1103/PhysRevD.96.113006. arXiv:1709.08217 [hep$\mathrm{ph}]$

53. W. Wang, Y.L. Shen, C.D. Lu, Phys. Rev. D 79, 054012 (2009). https://doi.org/10.1103/PhysRevD.79.054012. arXiv:0811.3748 [hep-ph]

54. R.H. Li, J.J. Hou, B. He, Y.R. Wang, https://doi.org/10.1088/ 1674-1137/abe0bc. arXiv:2010.09362 [hep-ph]

55. H.Y. Cheng, G. Meng, F. Xu, J. Zou, Phys. Rev. D 101(3), 034034 (2020). https://doi.org/10.1103/PhysRevD.101.034034. arXiv:2001.04553 [hep-ph]

56. H.Y. Cheng, arXiv:2109.01216 [hep-ph]

57. X.C. Zheng, C.H. Chang, T.F. Feng, X.G. Wu, Phys. Rev. D 100(3), 034004 (2019). https://doi.org/10.1103/PhysRevD.100. 034004. arXiv:1901.03477 [hep-ph]

58. A.J. Buras, arXiv:hep-ph/9806471

59. N. Brambilla, F. Karbstein, A. Vairo, Phys. Rev. D 87(7), 074014 (2013). https://doi.org/10.1103/PhysRevD.87.074014. arXiv:1301.3013 [hep-ph]

60. K.W. Wei, B. Chen, N. Liu, Q.Q. Wang, X.H. Guo, Phys. Rev. D 95(11), 116005 (2017). https://doi.org/10.1103/PhysRevD.95. 116005. arXiv:1609.02512 [hep-ph] 